\title{
A importância da fisioterapia imediata nos pós-operatório do ligamento cruzado
}

\section{anterior}

\author{
The importance of immediate physical therapy in the postoperative period of the anterior cruciate
} ligament

\section{La importancia de la fisioterapia inmediata en el postoperatorio del ligamento cruzado anterior}

Recebido: 06/01/2022 | Revisado: 10/01/2022 | Aceito: 12/01/2022| Publicado: 14/01/2022

\author{
Vera Lorena Galúcio Figueira \\ ORCID: https://orcid.org/0000-0002-9977-3905 \\ Fundação Esperança, Instituto Esperança de Ensino Superior, Brasil \\ E-mail: figueiragaluciosnap2@gmail.com \\ José Alexandre da Silva Júnior \\ ORCID: https://orcid.org/0000-0001-6600-7385 \\ Fundação Esperança, Instituto Esperança de Ensino Superior, Brasil \\ E-mail: josealexandre000@hotmail.com
}

\begin{abstract}
Resumo
O tratamento fisioterapêutico atua na reabilitação de pós-operatório da reconstrução do ligamento cruzado anterior (LCA), com a função de recuperar a mobilidade da articulação do joelho, reduzir o edema e o quadro álgico. São diversos os recursos e tratamentos utilizado na fisioterapia que podem contribuir para uma recuperação mais rápida do paciente. Esse estudo tem por objetivo expor e analisar a importância da fisioterapia imediata no pós-operatório de LCA, com intuito de comparar os métodos de tratamentos empregados na reabilitação de pós-operatório imediato de LCA, bem como, o conhecimento em âmbito científico do atendimento fisioterapêutico especializado em 48 horas, visando o bem-estar, a qualidade de vida e a prevenção. A metodologia aplicada nesta pesquisa segue as recomendações das revisões integrativas, a seleção dos artigos foi definida como critérios de inclusão: artigos publicados nos cinco últimos anos e artigos pesquisa de campo. Na busca foram excluídos artigos de revisões de literaturas, artigos que não abordavam a temática proposta, e pesquisa de comparação de técnicas interprofissionais. Em comum consenso mostram que a recuperação do paciente de reconstrução de LCA além de mais rápida, e mais eficaz com prognósticos mais favoráveis e previne a síndrome do imobilismo, lesões irreversíveis e calcificações. Desta forma conclui-se que a fisioterapia imediata e fundamental é eficaz nos pós cirúrgicos de LCA.
\end{abstract}

Palavras-chave: Fisioterapia imediata; Pós-operatório; LCA; Lesão.

\begin{abstract}
Physiotherapeutic treatment works in the post-operative rehabilitation of the reconstruction of the anterior cruciate ligament (ACL), with the function of recovering mobility in the knee joint, reducing edema and pain. There are several resources and treatments used in physical therapy that can contribute to a faster recovery of the patient. This study aims to expose and analyze the importance of immediate physiotherapy in the postoperative period of the ACL, in order to compare the treatment methods used in the immediate postoperative rehabilitation of ACL, as well as the knowledge in the scientific scope of specialized physiotherapy care in 48 hours, aiming at well-being, quality of life and prevention. The methodology applied in this research follows the recommendations of integrative reviews, the selection of articles was defined as inclusion criteria: articles published in the last five years and field research articles. The search excluded articles from literature reviews, articles that did not address the proposed theme, and research comparing interprofessional techniques. In common consensus, they show that the recovery of the patient after ACL reconstruction is not only faster, but also more effective, with more favorable prognoses, and prevents immobility syndrome, irreversible lesions and calcifications. Thus, it is concluded that immediate and fundamental physiotherapy is effective in post-operative ACL surgery.
\end{abstract}

Keywords: Immediate physiotherapy; Postoperative; LCA; Lesion.

\section{Resumen}

El tratamiento fisioterapéutico trabaja en la rehabilitación postoperatoria de la reconstrucción del ligamento cruzado anterior (LCA), con la función de recuperar la movilidad en la articulación de la rodilla, reduciendo el edema y el dolor. Existen varios recursos y tratamientos utilizados en fisioterapia que pueden contribuir a una recuperación más rápida del paciente. Este estudio tiene como objetivo exponer y analizar la importancia de la fisioterapia inmediata en el postoperatorio del ligamento cruzado anterior (LCA), con el fin de comparar los métodos de tratamiento utilizados en la rehabilitación postoperatoria inmediata de LCA, así como el conocimiento en el ámbito científico. de atención 
especializada en fisioterapia en 48 horas, con el objetivo de bienestar, calidad de vida y prevención. La metodología aplicada en esta investigación sigue las recomendaciones de revisiones integradoras, la selección de artículos se definió como criterios de inclusión: artículos publicados en los últimos cinco años y artículos de investigación de campo. La búsqueda excluyó artículos de revisiones de literatura, artículos que no abordaron el tema propuesto e investigaciones que compararon técnicas interprofesionales. En consenso común, muestran que la recuperación del paciente tras la reconstrucción del LCA no solo es más rápida, sino también más eficaz, con pronósticos más favorables, y previene el síndrome de inmovilidad, lesiones irreversibles y calcificaciones. Así, se concluye que la fisioterapia inmediata y fundamental es eficaz en el postoperatorio de la cirugía del LCA.

Palabras clave: Fisioterapia inmediata; Postoperatorio; LCA; Lesión.

\section{Introdução}

O joelho é uma das articulações com maior tendência a lesões nos ligamentos, encontrando-se no meio de dois grandes braços de alavanca, o fêmur e a tíbia, e por este motivo sofre maior número de forças rotacionais. Os ligamentos têm como principal função manter o equilíbrio do joelho como resposta as forças externas. Os ligamentos podem atuar de forma isolada ou relacionar-se com outros ligamentos o que facilita o estudo das principais causas da lesão (Duart \& Souza, 2017).

A lesão do Ligamento Cruzado Anterior (LCA), tipificada como entorse do joelho, trata-se da rotação interna do fêmur e rotação externa da tíbia. Os pacientes com entorse relatam ouvir um clique no joelho, seguido de forte dor incapacitante (Araújo \& Pinheiro, 2015).

O LCA tem sua origem na superfície posteromedial do côndilo femoral lateral e é inserido na tíbia na área intercondilar anterior. O rompimento do LCA leva à instabilidade da articulação do joelho, rotação interna excessiva e movimento anterior da tíbia, principalmente quando a última extensão é alcançada, resultando em atividades de vida diária restritas (Arliani et al., 2012; Soares et al., 2011).

Há evidencias que a doença musculoesquelética mais comum é aquela que atinge o joelho, sendo que o número de pacientes acometidos é de aproximadamente 4,8\% (48 a cada 1.000 pacientes). Aproximadamente 9\% das lesões do joelho envolvem o ligamento cruzado anterior (LCA). Em mais de 70\% dos casos, a lesão é causada por mecanismos sem contato, como desaceleração repentina e mudança de direção ou giro e / ou joelhos quase em linha reta após o salto de volta ao solo (Queiroz, 2018).

Nos Estados Unidos, aproximadamente 250.000 lesões do LCA ocorrem anualmente e aproximadamente 130.000 pessoas são submetidas à reconstrução do LCA. Essa cirurgia continua sendo o padrão ouro no tratamento das lesões do LCA, visto que aproximadamente $98 \%$ dos cirurgiões recomendam a reconstrução ligamentar para atletas que buscam retorno ao esporte. No entanto, apenas $65 \%$ retornam aos níveis de atividade pré-lesão e $55 \%$ aos níveis competitivos (Barroso \& Soares, 2020).

Com isso o papel da fisioterapia na reabilitação de pacientes submetidos à cirurgia de reconstrução do LCA é de extrema importância tanto na fase pré-operatória como na pós-operatória, uma vez que o fisioterapeuta cumpre os papéis de planejar e realizar o tratamento do paciente que sofreu esse tipo de lesão (Arliani et al., 2012).

Nas primeiras 48 horas do pós-operatório inicia-se a fisioterapia, e este procedimento tem por objetivo a prevenção de contraturas e deformidades, para a manutenção da integridade dos tecidos, além da proteção dos efeitos adversos da imobilização. Ela atua no controle da dor, melhora da amplitude de movimento (ADM) e na força muscular (Brumitt \& Jobst, 2015).

A escolha do tratamento fisioterapêutico depende da lesão, grau e extensão do trauma, podendo durar de seis meses a um ano, com diversos protocolos terapêuticos específicos para a fase vigente da lesão no momento da avaliação (Costa, 2016; Rosa et al., 2018; Andrade Filho et al., 2019; Carvalho et al., 2019; Barroso \& Soares, 2020).

A posposta desse estudo é comparar métodos no pós-operatório imediato de LCA, visto que sua utilização melhora os sinais e sintomas gerados para o procedimento cirúrgico. Dessa forma o presente trabalho tem por objetivo expor e analisar a importância da fisioterapia imediata no pós-operatório de LCA, com intuito de difundir conhecimento em âmbito científico do 
atendimento fisioterapêutico especializado em 48 horas, visando o bem-estar, a qualidade de vida e a prevenção de complicações.

\section{Metodologia}

Trata-se de uma revisão integrativa que foi realizada nas bases de dados Google Acadêmico, SciELO (Scientific Electronic Library Online), PubMed (National Library of Medicine and National Institutes of Health), BVS (Biblioteca Nacional de saúde), no período de novembro e dezembro de 2021. A estratégia de busca foi realizada a partir dos descritores: fisioterapia imediata, LCA - Ligamento Cruzado Anterior e lesão, organizados e apresentados na Figura 1.

Como critério de inclusão foram analisados artigos publicados entre 2011 a 2021 que abordassem a temática da importância da fisioterapia imediata no pós operatório de LCA. E como base de exclusão, artigos que eram revisões de literatura, ou que abordavam outras terapias, outras lesões, e que não eram estudos com humanos.

Foram incluídos 3 artigos e 2 ensaios clínicos randomizados onde os principais indicadores metodológicos dos estudos selecionados estão apresentados no Quadro 1.

Figura 1. Fluxograma de desenvolvimento da pesquisa.

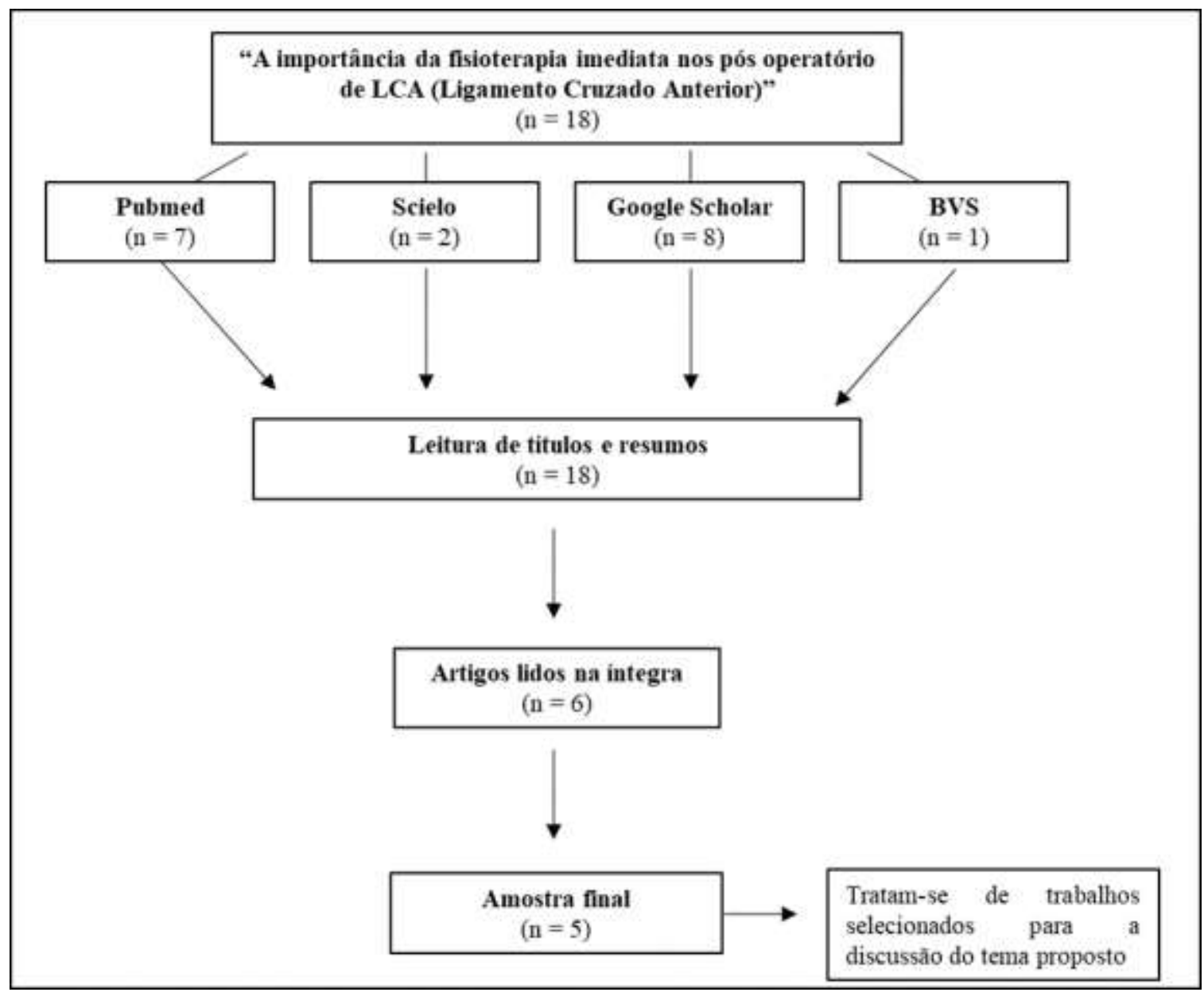

Fonte: Elaborado pelos próprios autores.

\section{Resultados}

$\mathrm{Na}$ busca realizada, foi utilizado o cruzamento de palavras chaves, como resultado foram encontrados 18 artigos. Destes artigos, 12 foram excluídos por se tratarem de dissertações, revisões ou por estarem incompletos, sobraram 6 artigos para leitura na íntegra. Logo após a leitura completa 1 artigo foi excluído devido não se enquadrar no perfil do estudo, restando apenas 5 artigos para a composição do banco de informações necessárias para o desenvolvimento do estudo como especifica o fluxograma 
da Figura 1, com o desdobramento do curso da pesquisa dos artigos para a revisão é possível visualizar as pesquisas realizadas entre 2011 e 2021, bem como informações contendo as seguintes características: autor, título do artigo, objetivo, metodologia, resultados e conclusões (Quadro 1).

Quadro 1. Trabalhos selecionados para a discussão do tema proposto.

\begin{tabular}{|c|c|c|c|c|}
\hline Autor/ano & Título & Objetivo & Metodologia & Conclusão \\
\hline Rosa et al., 2018 & $\begin{array}{l}\text { Avaliação e tratamento } \\
\text { fisioterapêutico no pós- } \\
\text { operatório de } \\
\text { reconstrução do } \\
\text { ligamento cruzado } \\
\text { anterior: relato de caso }\end{array}$ & $\begin{array}{l}\text { Descrever um relato de caso, de } \\
\text { um paciente que sofreu uma } \\
\text { reconstrução cirúrgica de LCA } \\
\text { e relatar a avaliação e } \\
\text { tratamento fisioterapêutico } \\
\text { desenvolvida com a paciente. }\end{array}$ & $\begin{array}{l}\text { O paciente, G.S.S., } 21 \text { anos, } \\
\text { militar, foi encaminhado ao } \\
\text { tratamento, no qual foi } \\
\text { realizado uma avaliação } \\
\text { fisioterapêutica e a partir do } \\
\text { diagnostico, foi elaborado o } \\
\text { plano terapêutico } \\
\text { adequado. }\end{array}$ & $\begin{array}{l}\text { Foi possível verificar a } \\
\text { redução da sintomatologia } \\
\text { álgica, assim como a marcha } \\
\text { independente sem uso do } \\
\text { dispositivo auxiliar. }\end{array}$ \\
\hline Queiroz, 2018 & $\begin{array}{c}\text { Predição da função e } \\
\text { índice de simetria por } \\
\text { meio de desfechos } \\
\text { clínicos e } \\
\text { neuromusculares em } \\
\text { indivíduos submetidos à } \\
\text { reconstrução do } \\
\text { ligamento cruzado } \\
\text { anterior } \\
\end{array}$ & $\begin{array}{l}\text { Identificar aspectos funcionais } \\
\text { e físicos que poderiam } \\
\text { redirecionar a intervenção e } \\
\text { melhor desfecho em relação a } \\
\text { essa população após a alta. }\end{array}$ & $\begin{array}{l}\text { Estudo transversal com } 34 \\
\text { indivíduos, } 18 \text { a } 40 \text { anos, } \\
\text { com ruptura do LCA no } 4^{\circ} \\
\text { mês de pós-operatório. }\end{array}$ & $\begin{array}{l}\text { Os resultados mostraram que } \\
\text { indivíduos que se encontram } \\
\text { no quarto mês de pós- } \\
\text { reconstrução do LCA } \\
\text { apresentam pior desempenho } \\
\text { tanto no membro acometido } \\
\text { como do membro não- } \\
\text { acometido. }\end{array}$ \\
\hline $\begin{array}{l}\text { Barroso \& } \\
\text { Soares, } 2020\end{array}$ & $\begin{array}{l}\text { Benefícios do tratamento } \\
\text { fisioterapêutico em um } \\
\text { paciente pós-operatório } \\
\text { de artroscopia em } \\
\text { menisco medial: relato de } \\
\text { caso }\end{array}$ & $\begin{array}{l}\text { O estudo teve como objetivo } \\
\text { principal apresentar um relato } \\
\text { de caso, descrevendo os } \\
\text { benefícios do tratamento } \\
\text { fisioterapêutico em um paciente } \\
\text { pós-operatório de artroscopia } \\
\text { em menisco medial. }\end{array}$ & $\begin{array}{l}\text { Paciente A.R.N.R.A. do } \\
\text { sexo feminino, } 49 \text { anos, } \\
\text { após um exercício no } \\
\text { crossfit, relatou dores no } \\
\text { joelho, onde foi } \\
\text { diagnosticada com uma } \\
\text { ruptura de menisco medial } \\
\text { esquerdo, logo depois, } \\
\text { realizou a artroscopia e } \\
\text { com } 9 \text { dias após a } \\
\text { intervenção cirúrgica } \\
\text { iniciou a fisioterapia pós- } \\
\text { operatória. }\end{array}$ & $\begin{array}{l}\text { A paciente realizou a } \\
\text { fisioterapia pós-operatória } \\
\text { imediata, sendo esses fatores } \\
\text { essenciais para permitir a } \\
\text { completa reabilitação } \\
\text { funcional. Portanto, sugere } \\
\text { realização de novos estudos } \\
\text { de caso com um número } \\
\text { maior de pacientes, com isso, } \\
\text { torna-se mais possível } \\
\text { comprar com os dados que } \\
\text { foram adquiridos no estudo. }\end{array}$ \\
\hline Rego et al., 2014 & $\begin{array}{l}\text { Atuação da fisioterapia } \\
\text { no pré e pós-operatório } \\
\text { de reconstrução do } \\
\text { ligamento cruzado } \\
\text { anterior (LCA) }\end{array}$ & $\begin{array}{c}\text { Investigar a atuação da } \\
\text { fisioterapia na reabilitação da } \\
\text { lesão do Ligamento Cruzado } \\
\text { Anterior. }\end{array}$ & $\begin{array}{l}\text { Foi feito uma seleção de } \\
\text { artigos publicados entre os } \\
\text { anos de } 2010 \text { a } 2013 \text { no } \\
\text { Lilacs, Pubmed, PEDro, } \\
\text { Scielo e no Google } \\
\text { acadêmico, utilizando-se } \\
\text { como descritores: } \\
\text { Ligamento Cruzado } \\
\text { Anterior e Fisioterapia, } \\
\text { Fisioterapia no pré e pós } \\
\text { operatório de Ligamento } \\
\text { Cruzado Anterior, Lesão de } \\
\text { Ligamento Cruzado } \\
\text { Anterior. } \\
\end{array}$ & $\begin{array}{l}\text { A atuação da fisioterapia na } \\
\text { lesão do Ligamento Cruzado } \\
\text { Anterior proporciona a } \\
\text { melhora da capacidade } \\
\text { funcional e adaptativa do } \\
\text { indivíduo em sua modalidade } \\
\text { esportiva e nas atividades de } \\
\text { vida diária, além de favorecer } \\
\text { a diminuição das sequelas. }\end{array}$ \\
\hline $\begin{array}{c}\text { Andrade } \text { et al., } \\
2019\end{array}$ & $\begin{array}{l}\text { Protocolo de reabilitação } \\
\text { no pós-operatório de } \\
\text { ligamentoplastia do } \\
\text { cruzado anterior do } \\
\text { joelho: estudo de caso }\end{array}$ & $\begin{array}{l}\text { Para tanto o objetivo do } \\
\text { presente estudo foi relatar o } \\
\text { estudo do caso de um paciente } \\
\text { diagnosticado com ruptura } \\
\text { completa do ligamento cruzado } \\
\text { anterior (LCA) de joelho direito } \\
\text { submetido a reconstrução do } \\
\text { LCA, atendido pelo serviço de } \\
\text { estágio da clínica Escola } \\
\text { Unifisio do curso de fisioterapia } \\
\text { da Uni Evangélica. }\end{array}$ & $\begin{array}{l}\text { Para coleta de dados foi } \\
\text { utilizado dados do } \\
\text { prontuário do paciente } \\
\text { contendo anamnese, exame } \\
\text { físico, objetivo e plano de } \\
\text { tratamento, bem como } \\
\text { evolução diária do mesmo. }\end{array}$ & $\begin{array}{l}\text { Observou-se que por meio do } \\
\text { tratamento proposto através } \\
\text { dos exercícios houve uma } \\
\text { evolução na melhora da } \\
\text { capacidade de deambulação e } \\
\text { na amplitude de flexo } \\
\text { extensão de joelho, } \\
\text { consequentemente } \\
\text { melhorando da qualidade de } \\
\text { vida do paciente. }\end{array}$ \\
\hline
\end{tabular}

Fonte: Elaborado pelos próprios autores. 


\section{Discussão}

No estudo realizado por Carvalho et al. (2019), diz que imediatamente após a lesão, deve ser dada atenção imediata à hemartrose e ao processo inflamatório geral. Exercícios de movimentação devem ser iniciados precocemente, para possibilitar a restauração da ADM e impedir complicações como fibrose na fossa intercondilar femoral e redução na força do quadríceps. Após o controle inflamatório inicial e restauração da ADM, devem ser iniciados exercícios de fortalecimento muscular. Os autores enfatizam a importância do tratamento do músculo quadríceps, tanto no pré-operatório, quanto no pós-operatório.

Relatam os autores o seguinte:

"A fase pós operatória foi iniciada ainda no hospital durante o período de internação com os objetivos, independente do paciente, foram: eliminar a dor, hemartorse, restaurar a ADM e iniciar exercício de fortalecimento músculo junto a progressão sensório motor (Carvalho et al., 2019).”

Em concordância Rêgo et al. (2014), diz que a fisioterapia na reabilitação no PO utiliza diferentes abordagens preconizando a funcionalidade do paciente e uma abordagem biopsicossocial, englobando desde controle da dor, edema e cicatriz, exercícios terapêuticos e treinos funcionais. Com isso, a terapia precoce gera resultados que trazem melhora para o paciente em menos tempo e também mudam o prognóstico do paciente para melhor.

Segundo Costa (2016), a prevenção dessas complicações deve ser o princípio básico de qualquer plano de tratamento, principalmente no tratamento, para obter bons resultados, deve-se iniciar o mais cedo possível. O tratamento precoce pode minimizar os efeitos negativos da imobilidade, proporcionar aos pacientes uma recuperação completa mais rápida, reduzir seu tempo de recuperação e melhorar sua qualidade de vida, mesmo após serem restritos. O ensaio randorizado de Costa foi composto por 44 voluntários do gênero masculino submetidos a reconstrução do LCA. Deste grupo 50\% fizeram uso da plataforma vibratória e os demais não fizeram o uso plataforma vibratória, porém o resultado foi o mesmo, não houve diferença relevante. Com isso a autora deixa claro, em sua pesquisa, que o tratamento deve ser ainda no período pós operatório (48 horas) pra que se tenha resultados relevantes, e que fora do período imediato o tratamento perde a eficácia.

O estudo de Andrade Filho e colaboradores (2019), mostra os resultados observados por meio da fisioterapia imediatamente após a reconstrução do LCA, e verificaram a redução dos sintomas de dor, a redução do edema e o aumento da amplitude de movimento do lado direito do joelho, bem como independência sem o uso de equipamentos auxiliares de caminhada. Este Relato de caso onde o paciente começou a fisioterapia uma semana após a cirurgia. $\mathrm{O}$ autor relata que após 8 atendimentos o paciente já apresentou melhora na macha, na extensão de joelho e na força muscular de que em extensão tem grau 4 e de flexão chaga a grau 5.

Até o momento, os resultados têm sido positivos, embora seja necessário tratamento contínuo para que o paciente alcance a recuperação funcional completa. A fase pós-operatória da reabilitação fisioterapêutica do LCA contralateral, o uso do exercício resistido isotônico unilateral é mais eficaz do que a forma bilateral para obter um maior grau de performance muscular entre os membros doador e receptor de simetria (Barroso \& Soares, 2020).

Nos trabalhos acima apresentados e discutidos, em comum consenso mostram que a recuperação do paciente de reconstrução de LCA é sim muito mais rápida e apresenta mais vantagens, como prevenir a síndrome do imobilismo, lesões irreversíveis, perca de força muscular, lesões que podem ser geradas pelo desuso e calcificações. Observar-se que o prognóstico de um paciente que inicia o tratamento fisioterapêutico até uma de pós operatório é muito mais eficaz do que a de um paciente que começa o tratamento até quatro semanas depois do procedimento cirúrgico.

\section{Conclusão}

Com esse estudo sugere-se que a fisioterapia imediata e fundamental é eficaz nos pós-cirúrgicos de LCA. Com a concordância do ponto de vista dos autores, há uma relevância significativa na reabilitação do paciente imediata desde a primeira 
sessão 48 horas após a cirurgia, com isso é possível acelerar o tempo de tratamento com a obtenção de resultados, a partir da primeira sessão, como diminuição de dor e edemas prevenido lesões resultantes por imobilização.

\section{Referências}

Aires, S. L. (2018). Reabilitação fisioterapêutica no pós-operatório imediato e tardio de lesões do manguito rotador. Trabalho de conclusão de curso. Faculdade de Educação e Meio Ambiente-Faema. Ariquemes.

Andrade Filho, A. L., Jayme, D. H. C., Leão, L. C. B., Souza, L. R. A., Vicentini, C. R., Zani, H. P., \& Costa, W. S. (2019). Protocolo de reabilitação no pósoperatório de ligamenroplástia do cruzado anterior do joelho: Estudo de caso. Revisão de literatura, Anais da XVI Mostra acadêmica do curso de fisioterapia. $7(7), 6$.

Araújo. A. G. S. \& Pinheiro, I. (2015). Protocolos de tratamento fisioterápico nas lesões de ligamento cruzado anterior após ligamentoplastia - Uma revisão. Cinergis. Joinvile, SC, Brasil. 16 (1), $61-65$.

Arliani, G. G., Astur, D. C., Michel, K., Kalela, C. C., \& Cohen, M. (2012). Lesão ligamento cruzado anterior fazer: Tratamento e Reabilitação. Perspectivas e Tendências atuais. Rev. bras. ortop., 47(2).

Barroso, A. K. S. \& Soares, J. S. (2020). Benefícios do tratamento fisioterapêutico em um paciente pós-operatório de artroscopia em menisco medial: relato de caso Brazilian Journal of Development.Braz. J. of Develop., Curitiba. 6(9), 70080-70095.

Brumitt, J., \& Jobst, E. E. (2015). Casos clínicos em fisioterapia ortopédica. AMGH.

Carvalho, J. P. O., Lima, R. N., \& Oliveira, M. P. (2019). Estratégias de aplicação do exercício resistido no pós-operatório do lca contralateral. Relatório final de pesquisa de Iniciação Científica apresentado à Assessoria de Pós-Graduação e Pesquisa., Centro Universitário De Brasília - UniCEUB Programa De Iniciação Científica. p. 81.

Costa, K. S. A. (2016). Efeitos imediatos na plataforma vibratória no desempenho neuromuscular do quadríceps femoral após reconstrução de LCA: Ensaio controlado, Randomizado e cego. Dissertação apresentada ao programa de pós graduação de fisioterapia da Universidade Federal do Rio Grande do Norte. p. 61 .

Duart, A. D. \& Souza, F. L. P. (2017). Reabilitação no pós-operatório de ligamento cruzado anterior através de cinesioterapia associada à eletroterapia. Pósgraduação em Reabilitação em Ortopedia e Traumatologia com ênfase em Terapia Manual Faculdade Faserra.

Freitas, E. M. (2019). Eletroestimulação E Cinesioterapia Para Aplicabilidade Clínica Na Lesão De Ligamento Cruzado Anterior. Estudo, Caderno De Pesquisa Aplicada 1(1), julho-dezembro de 2019 - Instituto Superior de Ciências Aplicadas (Limeira - SP). p. 17.

Leão, M. G. D. S. (2017). Controle da dor pós-operatória em pacientes submetidos à reconstrução do ligamento cruzado anterior do joelho: comparação de soluções analgésicas intra-articulares.

Matos, L. M. R. S., Dos Santos, R; M. E., Medrado, B. B., Lessa, E. A., Bezerra, N. K. M. S., \& Bonfim, E. M. S. (2021). Fisioterapia no pós-operatório de artroplastia de quadril: uma revisão sistemática. Fisioter. Bras. 21(6), 609-618.

Nascimento, E. S. B. (2018). Uso da cinesioterapia no tratamento fisioterapêutico em idoso após fratura de fêmur. Monografia, FAEMA. p. 33.

Nascimento, M. N. (2021). Impactos da acupuntura no tratamento de pós-operatório do ligamento cruzado anterior em jogadores de futebol. Research, Society and Developmen. 10(7).

Noia, A. L. F., Alves, S. S., \& Milcent, E. N. R. (2021). Efeitos Da Cinesioterapia Em Pacientes No Pós-Operatório De Reconstrução Do Ligamento Cruzado Anterior (LCA): Revisão de literatura, Revista Ibero-Americana de Humanidades, Ciências e Educação. São Paulo. 7 (8). doi.org/ 10.51891/rease.v7i8.2024, 874 ,

Oliveira, G. B. (2017). A intervenção fisioterapêutica em lesões do ligamento cruzado anterior em atleta de voleibol. Trabalho de conclusão e curso. 34.

Queiroz, N. C. A. (2018). Predição da função e índice de simetria por meio de desfechos clínicos e neuromusculares em indivíduos submetidos à reconstrução do ligamento cruzado anterior. Programa De Pós-Graduação Em Ciências Da Reabilitação. p.103.

Rêgo, A. S., Salgado, f. H. S., Santos, H. P., Aragão, M. B., \& Pereira, S. K. (2014). Atuação da Fisioterapia Pre e pós-operatório de reconstrução do ligamento cruzado anterior (LCA). Revista Brasileira de Prescrição e Fisiologia do Exercício. 8 S1(46). 341.

Reis, D. T. F. (2019). O tratamento fisioterapêutico através da crioterapia em lesões de ligamento cruzado anterior em mulheres praticantes de Jiu-Jitsu.Braz. J. Hea. Rev., 2(1), 7.

Rosa, N. Q., Cattelan, A. V., Freitas, A. C. S., Teixeira, L. P., \& Lara, S. (2018). De Participação: Iniciação Científica Avaliação e Tratamento Fisioterapêutico no Pós-Operatório de Reconstrução do Ligamento Cruzado Anterior: Relato de Caso. Intervenção Fisioterapêutica em Lesão do Ligamento Cruzado Anterior em Atletas de Voleibol, Anais do 10 Salão Internacional de Ensino, Pesquisa e Extensão - SIEPE. p. 6.

Santiago, A. S. \& Joia, L. C. (2018). Benefícios Do Treino De Marcha Em Pacientes Com Pós-Operatório De Ligamento Cruzado Anterior (LCA). Artigo, Revista das Ciências da Saúde do Oeste Baiano - Higia. 3. 14.

Santos, A. M. R., Pereira, D. B. D., Carvalho, L. C. S., Madeira, M. C. A., \& Andrade, E. M. L. R. (2016). Acidentes Domésticos em idosos atendidos em um Hospital de urgência. Rev. Eletr. Enf. 
Research, Society and Development, v. 11, n. 1, e52111125450, 2022

(CC BY 4.0) | ISSN 2525-3409 | DOI: http://dx.doi.org/10.33448/rsd-v11i1.25450

Santos, T. H. M. (2016). Protocolos de tratamento fisioterapêutico no pós operatório de reconstrução do ligamento cruzado anterior em atletas profissionais: revisão de literatura. Revista Científica FacMais VII(3).

Santos, A. F.; \& Vieira, K. V. S. (2021). Eficácia da fisioterapia na manutenção da capacidade funcional de idosos pós cirurgia de fratura proximal de fêmur. Revista Ibero-Americana de Humanidades, Ciências e Educação. São Paulo. 7(9).

Santos, D. T. (2021). Intervenção fisioterapêutica em pacientes com lesão do ligamento cruzado anterior: uma revisão integrativa. Monografia.

Silva, K. N. G., Imoto, A. M., Cohen, M., \& Peccin, M. S. (2010). Reabilitação Pós-Operatória Dos Ligamentos Cruzado Anterior E Posterior - Estudo De Caso. Ostoperative rehabilitation of the anterior and posterior cruciate ligaments - CASE STUDy, 1 - Universidade São Marcos / Instituto Cohen - Ortopedia, Reabilitação e Medicina do Esporte. 2 - Universidade Federal de São Paulo / Escola Paulista de Medicina (UNIFESP/EPM).

Silva, L. R. (2020). Pós operatório de lesão de ligamento cruzado anterior (LCA): Uma revisão dos métodos empregados na reabilitação. Trabalho de conclusão de curso, FAEMA. p. 34.

Soares M. S., Marques, R. L., Frazão, R. S., \& Assis, T. O. (2011). Intervenção fisioterapêutica no pós-operatório de lesões do ligamento cruzado anterior. TEMA - Revista Eletrônica de Ciências. 